\title{
MICROMACHINED OBLIQUE INCIDENCE REFLECTOMETRY (OIR) PROBE FOR SKIN CANCER DETECTION
}

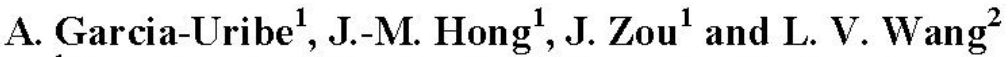 \\ ${ }^{1}$ Department of Electrical and Computer Engineering, \\ Texas A\&M University, College Station, TX 77843, USA \\ ${ }^{2}$ Department of Biomedical Engineering, \\ Washington University, St. Louis, MO 63130, USA \\ (Tel: +1-979-862-1640; E-mail: junzou@ece.tamu.edu)
}

\begin{abstract}
We present the design, fabrication and in-vivo testing of a new micromachined probe for skin cancer detection based on oblique incidence reflectometry (OIR). The device miniaturization and fabrication precision provided by micromachining ensure reliable and repeatable high performance of the probe. The developed probe has been tested in a pre-clinical condition. An overall sensitivity of $100 \%$ and specificity of $93 \%$ haven been achieved in the classification of malignant and benign skin lesions.
\end{abstract}

Keywords: Micromachined probe, diffuse reflectance, spectroscopy, skin cancer

\section{INTRODUCTION}

Skin cancer is the most common form of cancer and melanoma is the most serious and fatal type of skin cancer. According to the American Cancer Society, there are more than 62,000 new cases of melanoma and $1,000,000$ cases of nonmelanoma skin cancer with 8,000 deaths in the U.S. each year $[1,2]$. For most skin cancers, early diagnosis is the key for a successful treatment. Oblique incidence reflectometry (OIR) is a novel non-invasive optical detection method, which can be utilized to classify skin tumors based on their optical diffuse reflectance [3]. Previous studies have shown that OIR is capable of effectively differentiate carcinoma skin cancer lesion and precancerous dysplastic nevus from benign ones [4].

To conduct OIR measurement, a special optical fiber probe has to be constructed and used to capture the optical diffuse reflectance spectra of the targeted skin lesion. The optical sensor probe consists of source fibers (for light incidence) and collection fibers (for reflected light from skin lesion). Both the source fibers and collection fibers need to be precisely aligned to each other, which can be achieved with a mechanical positioning device (Fig. 1). The entire sensor probe has to be as small as possible in order to detect even the early-stage skin cancer lesions (usually smaller than $3 \times 3 \mathrm{~mm}^{2}$ ). However, it is extremely difficult and costly to fabricate the mechanical position device using conventional machining process.

In this paper, we present the development of a new OIR probe for skin cancer detection, which is fabricated with micromachining technology. The micron-size precision and mass production capability of micromachining ensures the needed alignment accuracy of the optical fibers, as well as the compact size and low cost fabrication of the probe.

\section{PROBE DESIGN AND FABRICATION}

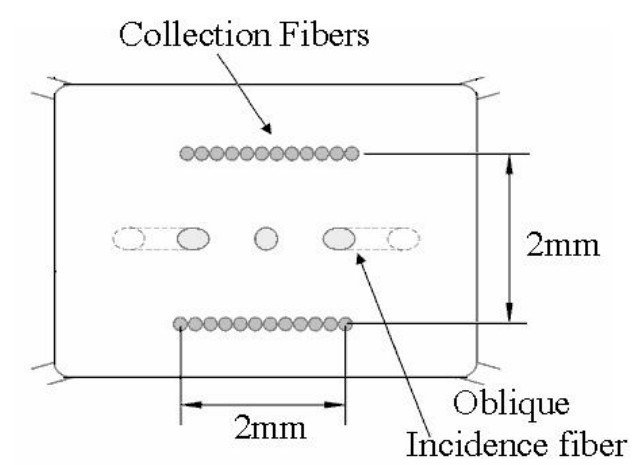

Fig. 1 Front view of the OIR probe design.

The design of the OIR probe is shown in Fig. 1, which includes three source fibers (each with a diameter of $200 \mu \mathrm{m}$ ) and two groups of twelve collection fibers (each with a diameter of $100 \mu \mathrm{m}$ ) within an area of $2 \times 2 \mathrm{~mm}^{2}$. To achieve accurate 
alignment of the source and collection fibers necessary for robust clinical application, four micromachined positioning devices (two for source fibers and two for collection fibers) were fabricated. Each positioning device consists of a silicon substrate with photolithography patterned SU-8 guiding structures (Fig. 2a). The thickness of the SU-8 layer is $100 \mu \mathrm{m}$. After assembling both source and collection fibers into the SU-8 guiding structures, all the positioning chips are stacked and glued together with epoxy (Fig. $2 b$ and 2c). Among the three source fibers, one is used for normal incidence and the other two for oblique incidence. Two positioning devices are assembled face to face to accommodate the source fibers of $200 \mu \mathrm{m}$ in diameter. Finally, the assembled probe is mounted into an aluminum probe holder to facilitate the testing (Fig. 2d).

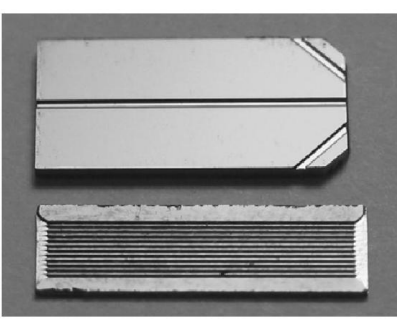

(a)

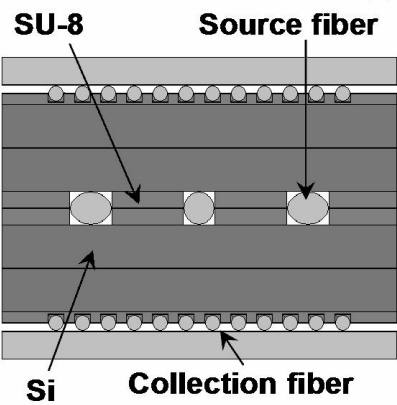

(c)

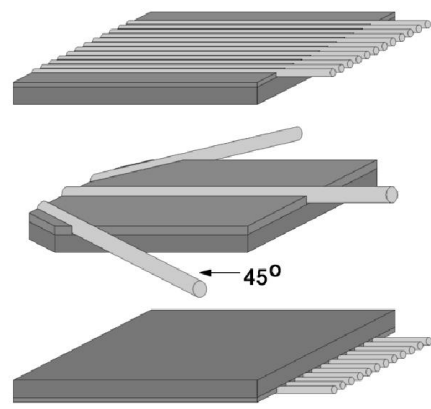

(b)

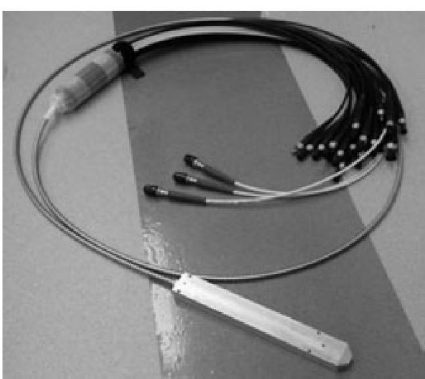

(d)
Fig. 2 OIR probe fabrication and assembly.

\section{EXPERIMENTAL SETUP AND CALIBRATION}

The testing set-up of the OIR is shown in Fig 3 A halogen lamp is used as the light source. The optical multiplexer allows the light delivery through only one source fiber at a time to the area of interest. Once delivered to the skin, the incident light interacts with the medium (skin lesion), whose diffuse reflectance is then collected by the collection fibers. The collection fibers are coupled with the imaging spectrograph that generates an optical spectrum for each fiber. The CCD camera collects the spectral-images from the wavelength range from 455 to $765 \mathrm{~nm}$. The spectral images represent the steady-state diffuse reflectance spectra from each collection fiber, which are stored in a personal computer for further analysis.

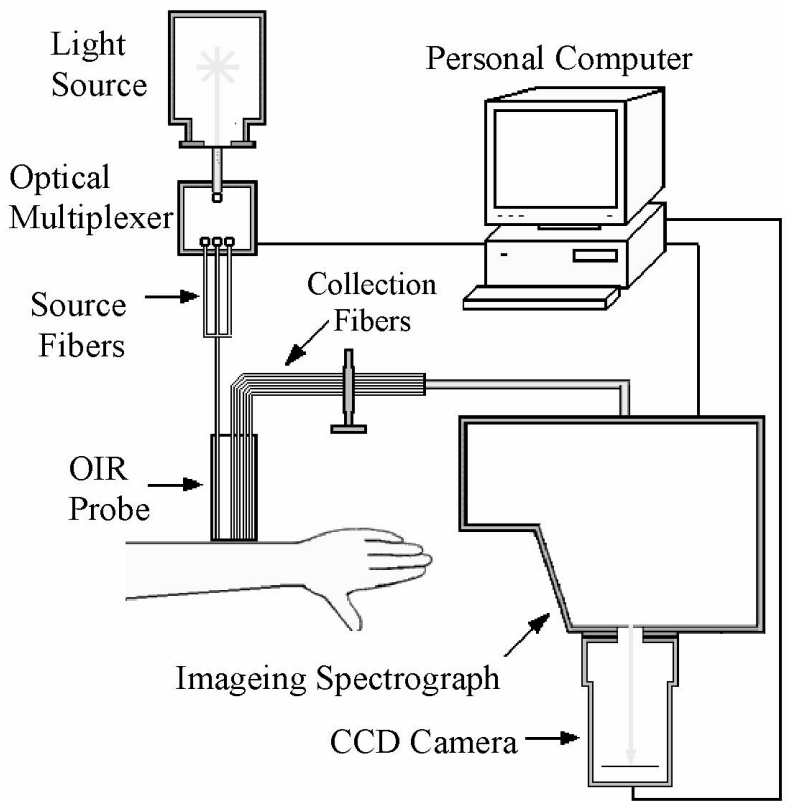

Fig. 3 OIR test setup.

Before the OIR measurement, the entire set-up was first calibrated using a liquid reference solution, consisting of trypan blue dyes as the absorbers and polystyrene microspheres as the scatterers [3,5]. Fig. 4 shows the expected and the estimated optical spectral properties (absorption coefficient $\left(\mu_{\mathrm{a}}\right)$ and reduced scattering coefficient $\left(\mu_{\mathrm{s}}{ }^{\prime}\right)$ of the reference solution. The expected properties were calculated based on the diffusion theory of diffuse reflectance [6] and an equation previously developed for obliqueincidence reflectometry [7]. The estimated properties were obtained using diffusion theory and scaleable Monte-Carlo to match the measured steady state diffuse reflectance for each wavelength $[3,8]$. The calibration of the system is deemed to be complete when the difference between the expected and estimated values of $\mu_{\mathrm{a}}$ and $\mu_{\mathrm{s}}{ }^{\prime}$ is small enough (e.g. $<2 \%$ in Fig. 4). 
Absorption spectra $\left(\mu_{\mathrm{a}}\right)$

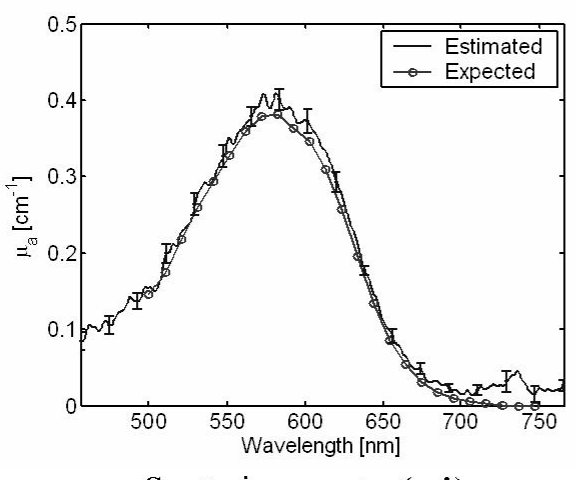

Scattering spectra $\left(\mu_{\mathrm{s}}{ }^{\prime}\right)$

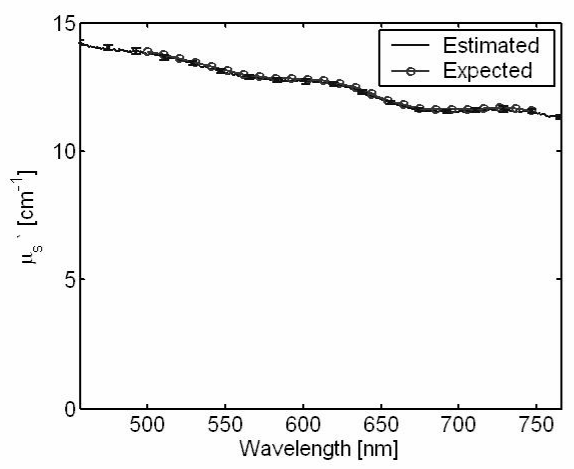

Fig. 4 Expected (from simulation) and estimated (from measurement) absorption and scattering spectra of the liquid reference solution for OIR system calibration.

\section{IN-VIVO TESTING}

The developed probe was used to collect the steady-state spatially resolved diffuse reflectance from human skin. Fig. 5 shows a spectral-image from healthy human skin. The absorption spectra $\left(\mu_{\mathrm{a}}\right)$ and scattering spectra $\left(\mu_{\mathrm{s}}^{\prime}\right)$ were then estimated, which are shown in Fig. 6.

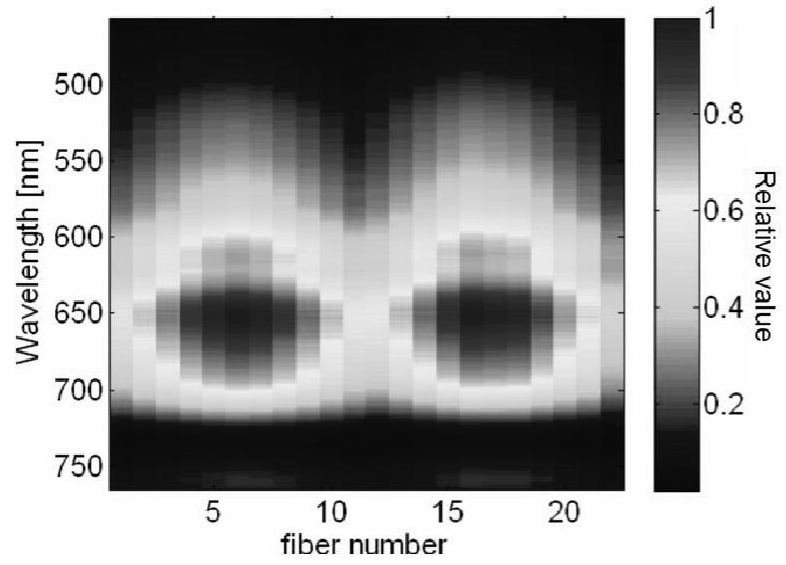

Fig. 5 Spectral Image from normal human skin.
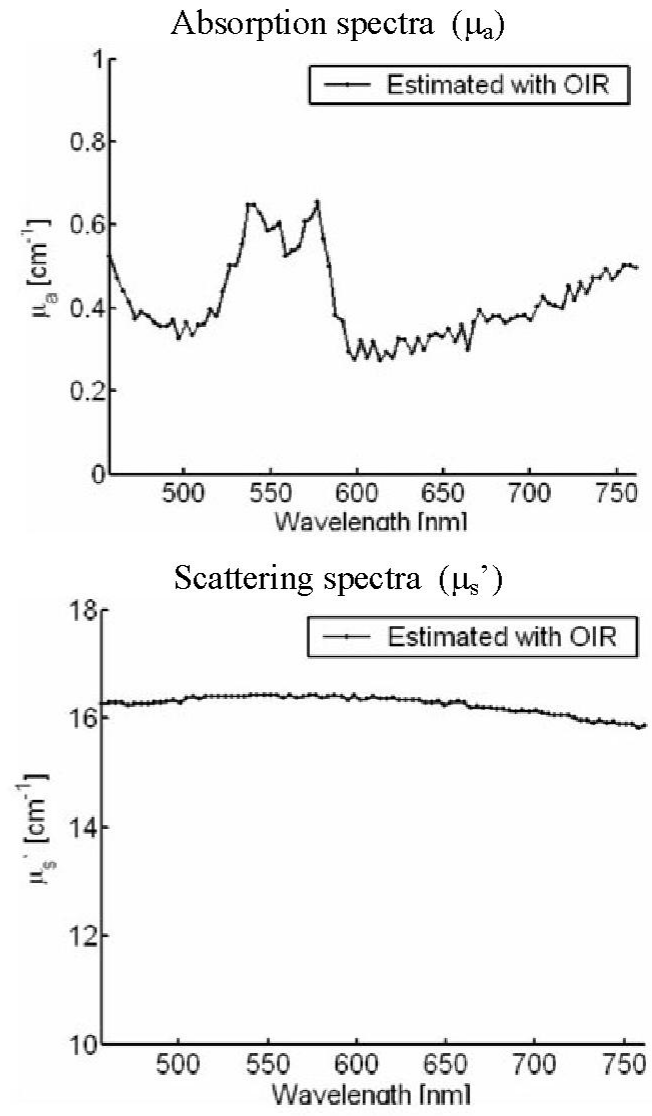

Fig. 6 Estimated absorption and scattering spectra of normal human skin.

For the pre-clinical testing of the developed OIR probe, the diffuse reflectance spectra were collected from 111 pigmented skin lesion including 15 histopathologically diagnosed as malignant melanoma or severe dysplastic nevus. Each spectral image from a lesion was normalized by a spectral image collected form the surrounding healthy tissue. This is based on the assumption that the melanin content of healthy skin tissue will correlate with that of the imaged lesion.

A software program was developed for skin cancer classification based on the collected normalized spatially resolved diffuse reflectance spectra [4]. The absorption spectra $\left(\mu_{a}\right)$ and scattering spectra $\left(\mu_{\mathrm{s}}{ }^{\prime}\right)$ were also estimated, which will be used to study their correlation with the physical/physiological properties of the skin lesions in the future. Among the 111 test data, the first 67 were used for the training of the classification program to differentiate malignant melanoma and severe dysplastic nevus from 
moderate and mild dysplastic nevus, common nevus, actinic and seborrheic keratoses. The remaining 44 data were then used for testing.

As shown in Table 1 and 2, the classification program successfully distinguishes malignant from benign lesions with an overall accuracy of $93 \%$ and $98 \%$ for the training and testing sets, respectively. The sensitivity is $100 \%$ for both training and testing sets with a specificity of $91 \%$ and $97 \%$, respectively. Sensitivity indicates the fraction of correctly identified positive cases over all positive (malignant) cases. Specificity indicates the fraction of correctly identified negative cases over all negative (benign) cases.

Table 1. Classification performance for training data set.

\begin{tabular}{|l|c|c|c|}
\hline & Malignant & Benign & Correct \\
\hline Malignant & 12 & & $100 \%$ \\
\hline Benign & 5 & 50 & $91 \%$ \\
\hline
\end{tabular}

Table 2. Classification performance for testing data set.

\begin{tabular}{|l|c|c|c|}
\hline & Malignant & Benign & Correct \\
\hline Malignant & 3 & & $100 \%$ \\
\hline Benign & 1 & 40 & $97 \%$ \\
\hline \multirow{2}{*}{} & Overall & $98 \%$ \\
\cline { 2 - 4 }
\end{tabular}

\section{CONCLUSIONS}

A new micromachined OIR sensor probe has been developed for non-invasive detection of skin cancers. Micromachining plays a significant role in the probe development by enabling device miniaturization, low-cost fabrication and precision assembly, which cannot be matched by conventional machining techniques. By further reducing the probe size and adding new functionality, OIR could be readily expanded to the diagnosis of more treacherous epithelial cancers inside human bodies. The preliminary results indicate that the approach presented can potentially be used for computer-assisted early detection technique of skin cancer.

\section{ACKNOWLEDGMENTS}

The authors thank Dr. Duvic, Dr. Mays, Dr. Hymens and Dr. Mansfield for their help during the data collection at The University of Texas M.D. Anderson Cancer Center (Melanoma and Skin Center). This project is supported by National Institutes of Health under contract R01 CA106728.

\section{REFERENCES}

[1] American Cancer Society website. Melanoma and Non-melanoma Skin Cancer, http://www.cancer.org.

[2] Cancer Facts \& Figures 2006, American Cancer Society, 2006.

[3] G. Marquez and L. H. Wang, "White light oblique incidence reflectometer for measuring absorption and reduced scattering spectra of tissue-like turbid media," Optics Express, vol. 1, pp 454-460, 1997.

[4] A. Garcia-Uribe, N. Kehtarnavaz, G. Marquez, V. Prieto, M. Duvic, and L. V. Wang, "Skin cancer detection by spectroscopic oblique-incidence reflectometry: classification and physiological origins," Applied Optics, vol. 43, pp 2643-2650, 2004.

[5] H. Van de Hulst, Light Scattering by Small Particles, Dover Publications, New York, 1983.

[6] T. J. Farrell, M. S. Patterson, B. C. Wilson, "A diffusion theory model of spatially resolved, steady-state diffuse reflectance for the noninvasive determination of tissue optical properties in vivo," Medical Physics, vol. 19, pp 879-888, 1992.

[7] L.-H. Wang and S. L. Jacques, "Use of a laser beam with an oblique angle of incidence to measure the reduced scattering coefficient of a turbid medium," Applied Optics, vol. 34, pp 2362-2366, 1995.

[8] A. Kienle and M. S. Patterson, "Determination Of the Optical Properties Of Turbid Media From a Single Monte Carlo Simulation," Physics in Medicine and Biology, vol. 41, pp 2221-2227, 1996. 